\title{
Litiasi renale: prevenzione e terapia
}

\author{
Teresa Arcidiacono, Giulia Magni, Lorenza Macrina \\ e Giuseppe Vezzoli
}

journals.sagepub.com/home/gtn

\begin{abstract}
Nephrolithiasis: prevention and therapy

Recent guidelines have identified two steps in the prevention of recurrent urinary stones. The first includes general measures that may be recommended in all patients, irrespective of stone composition. These recommendations are substantially nutritional and include a diet with balanced intake of nutrients and abundant fluid ingestion, low in salt and normal in caloric intake, rich in vegetables and including dairy products. The second step includes specific measures based on the clinical activity of the kidney stone disease and the presence of acquired or hereditary conditions favouring recurrent stone formation. In this setting clinicians may prescribe drugs according to the composition of the stones and the urinary or metabolic abnormalities associated with the stones; surgical treatment may be coupled with medical therapy when there is malformation of the urinary tract or infected stones. Therefore, the role of clinicians in the treatment of stone formers is complex and requires the ability to characterize patients from a clinical, metabolic and nutritional point of view in order to provide the most personalized treatment possible.
\end{abstract}

\section{Keywords}

Nephrolithiasis, cystinuria, hypercalciuria, hypocitraturia, hyperuricuria, hyperossaluria

\section{Introduzione}

Dal 2010 sono state pubblicate quattro linee guida per il trattamento della calcolosi renale redatte a cura del Gruppo Italiano di Studio Multidisciplinare per la Calcolosi Renale, ${ }^{1}$ della European Association of Urology (EAU), ${ }^{2}$ della American Urology Association (AUA) ${ }^{3}$ e della American College of Physicians (ACP). ${ }^{4}$ Con il presente lavoro abbiamo cercato di sintetizzare i provvedimenti necessari per la prevenzione delle recidive di nefrolitiasi alla luce di quanto indicato in queste linee guida e anche considerando i criteri d'intervento che sono comunemente adottati nella pratica clinica. Sono stati perciò distinti due livelli di intervento terapeutico, uno più generale, che può essere intrapreso in tutti i pazienti indipendentemente dal tipo di calcolosi ed un secondo livello, più specifico, che può essere prescritto in base alle caratteristiche cliniche della malattia, al tipo di calcolo e ai fattori di rischio di recidiva.

\section{Le misure preventive generali}

Le diverse linee guida concordano nel definire una serie di provvedimenti generali che possono essere utili per la prevenzione della nefrolitiasi. Sono provvedimenti efficaci nelle diverse forme di calcolosi, indipendenti dalla composizione dei calcoli e di carattere sostanzialmente alimentare (tabella 1) tra i quali vi è anche la necessità di mantenere il peso corporeo ideale.

Il primo e fondamentale provvedimento è l'aumento dell'assunzione di fluidi; 5 l'obbiettivo è di produrre almeno 2-2,5 L di urine al giorno con un peso specifico minore di 1010. Bisogna perciò motivare i pazienti affinché facciano attenzione al volume urine più che al bere in sé e perché consumino bevande non dolcificate distribuendone l'assunzione in modo continuativo durante la giornata, bevendo anche prima di coricarsi e durante la notte quando si alza per andare in bagno.

Anche la dieta iposodica si è dimostrata utile nella riduzione delle recidive di calcolosi perché diminuisce l'escrezione delle sostanze litogene, come la cistina, il calcio e l'acido urico. ${ }^{6}$ L'apporto di sodio è in larga parte

Unità di Nefrologia e Dialisi, IRCCS, Istituto Scientifico San Raffaele e Università Vita Salute San Raffaele, Milano, Italy

\section{Corresponding author:}

Giuseppe Vezzoli, Unità di Nefrologia e Dialisi, IRCCS Istituto

Scientifico San Raffaele, Via Olgettina 60, Milano 20I32, Italy.

E-mail: vezzoli.giuseppe@hsr.it 
Tabella I. Misure generali preventive della nefrolitiasi.

Assunzione di fluidi

Dieta bilanciata
Bere 2,5-3 $L$ di fluidi al giorno

Distribuire l'assunzione di fluidi nelle 24 ore

Evitare di bere bevande zuccherate

Mantenere la diuresi di 2-2,5 L al giorno

II peso specifico delle urine deve essere inferiore a 1010

Supplire le perdite di fluidi (p. es. in caso di ipersudorazione o febbre) determinato dall'assunzione di sale da cucina $(\mathrm{NaCl})$ e può essere stimato con la misurazione del sodio e del cloro nelle urine di 24 ore. Le linee guida italiane raccomandano di consumare quantità di $\mathrm{NaCl}$ inferiori $\mathrm{ag} / \mathrm{die}$, corrispondenti a $100 \mathrm{mmol} / \mathrm{die}$, mentre le linee guida della EAU sono più severe e raccomandano un consumo di $\mathrm{NaCl}$ inferiore a $5 \mathrm{~g}$ / die, cioè minore di $80 \mathrm{mmol} /$ die di sodio o cloro. ${ }^{1,2}$

La dieta povera di calcio è stata per diversi anni in pas sato consigliata per prevenire le recidive di calcolosi; a tale scopo veniva perciò drasticamente diminuito il consumo dei latticini. Tuttavia, studi epidemiologici e d'intervento hanno osservato che la dieta povera di calcio/latticini favoriva lo sviluppo di calcoli renali, oltre che di osteoporosi. ${ }^{7,8}$ I moderni orientamenti propongono perciò che anche i soggetti calcolotici mantengano la loro assunzione di calcio tra 1 e 1,3 g/die in relazione all'età, al sesso e allo stato dell'individuo, come raccomandato da diversi istituti in funzione della massa ossea (https://www. niams.nih.gov/health info/bone/bone health/nutrition). La dieta priva di latticini apporta mediamente circa $400 \mathrm{mg} / \mathrm{die}$ di calcio $;{ }^{9}$ il contributo dei latticini è perciò fondamentale per rispettare l'assunzione raccomandata di calcio. Nel latte vaccino, sia scremato che intero, sono contenuti circa 120 $\mathrm{mg}$ di calcio per $100 \mathrm{ml}$ di prodotto ed è perciò sufficiente bere $500 \mathrm{ml}$ di latte al giorno perché l'assunzione dietetica totale di calcio sia pari a $1 \mathrm{~g} / \mathrm{die}$. Lo stesso risultato può essere ottenuto con $50 \mathrm{~g} / \mathrm{die}$ di grana o parmigiano o groviera. La tabella 2 mostra il contenuto medio di calcio degli alimenti che ne sono più ricchi. In alternativa ai latticini, le acque minerali (intese come acque nonoligominerali) possono essere un utile strumento per normalizzare l'assunzione di calcio; le acque minerali disponibili nel commercio possono contenere da 300 a 600 mg di calcio per L e l'assunzione di 1-2 L permette di raggiungere l'assunzione di calcio raccomandata. Bisogna però avere l'accortezza di bere queste acque principalmente ai pasti perché i supplementi di calcio assunti al di fuori dei pasti sembrano favorire lo sviluppo di calcolosi renale. ${ }^{7}$ Altri cibi che possono aumentare l'assunzione di calcio sono le mandorle, i ceci, il pesce azzurro, i crostacei, la rucola (tabella 2), ma ci sembrano però meno proponibili per raggiungere l'apporto raccomandato di calcio. La funzione "antilitogena" del calcio alimentare sembra essere spiegata dalla sua capacità di legare l'ossalato nel lume intestinale impedendone così l'assorbimento. Il calcio assunto al di fuori dei pasti non può legare l'ossalato e viene assorbito aumentando il potenziale rischio litogeno. ${ }^{7}$

Studi epidemiologici hanno osservato come un elevato consumo di carne fosse associato allo sviluppo di calcolosi renale; viceversa, il rischio litogeno risultava progressivamente ridotto nei soggetti che consumavano pesce e vegetali. ${ }^{10}$ Sia il consumo delle carni rosse (bovino e maiale) che bianche (pollame) accentuavano il rischio litogeno che cresceva con l'aumentare delle quantità di carni ingerite nelle popolazioni studiate. ${ }^{11,12}$ Questi dati indicano come la dieta più corretta per la prevenzione della calcolosi debba limitare il consumo delle carni ed incrementare quello dei vegetali trai quali i legumi si caratterizzano per un elevato contenuto di proteine. ${ }^{12}$ I moderni criteri di prevenzione della calcolosi raccomandano perciò un consumo di $0,8-1 \mathrm{~g} / \mathrm{kg}$ die di proteine animali. È stato supposto che le proteine di origine animale possano incrementare il carico di acidi della dieta e che questo possa agire sul tubulo renale e sull'osso causando l'aumento dell'escrezione di calcio e la riduzione di quella di citrato. Alcuni studi hanno però smentito questa ipotesi e hanno dimostrato che le proteine animali possono stimolare la produzione renale dell' $1,25(\mathrm{OH})_{2} \mathrm{D}$ con conseguente aumento dell'assorbimento intestinale di calcio e della sua escrezione. ${ }^{13,14}$

I cibi di derivazione animale arricchiscono di acidi l'organismo a causa delle caratteristiche biochimiche delle proteine contenute nella carne e nei latticini. I vegetali sono invece cibi che garantiscono l'apporto delle basi. Fanno eccezione a questa regola i farinacei, come la pasta e il pane, che contengono però quantità di acidi minori rispetto agli alimenti di origine animale. ${ }^{15}$ L'assunzione dietetica netta di acidi, inteso come differenza tra l'apporto nutrizionale di acidi a di basi, può essere positivo o negativo nel caso che prevalgano l'apporto degli acidi su quello delle basi o 
Tabella 2. Contenuto di calcio dei diversi alimenti espresso in milligrammi per $100 \mathrm{~g}$ di alimento.

\begin{tabular}{|c|c|c|}
\hline Tipo di alimento & Alimento & Contenuto di calcio $(\mathrm{mg} / \mathrm{l} 00 \mathrm{~g})$ \\
\hline \multirow[t]{2}{*}{ Latte } & Latte di capra & $|4|$ \\
\hline & Latte di vacca intero o scremato & $119-125$ \\
\hline \multirow[t]{2}{*}{ Yogurt } & Yogurt greco da latte intero & 150 \\
\hline & Yogurt scremato o intero & $120-135$ \\
\hline \multirow[t]{8}{*}{ Formaggi } & Grana, parmigiano, emmenthal, groviera & $1130-1160$ \\
\hline & Fontina, caciocavallo, provolone & $720-870$ \\
\hline & Pecorino, stracchino, brie, caciotta toscana, scamorza & $512-607$ \\
\hline & Taleggio, gorgonzola, crescenza & $400-433$ \\
\hline & Caciottina, camembert, ricotta di bufala, ricotta di vacca & $290-360$ \\
\hline & Mozzarella di bufala & 210 \\
\hline & Ricotta di pecora, fior di latte, mozzarella di vacca & 166 \\
\hline & Formaggio cremoso spalmabile & 110 \\
\hline \multirow[t]{9}{*}{ Non latticini } & Mandorle secche & 240 \\
\hline & Nocciole e noci secche & $140-150$ \\
\hline & Rughetta o rucola & 309 \\
\hline & Spinaci surgelati & 170 \\
\hline & Cicoria da taglio & 150 \\
\hline & Ceci e fagioli secchi (crudi) & $130-142$ \\
\hline & Sgombro & 185 \\
\hline & Acciuga o alice fresca & 148 \\
\hline & Molluschi freschi & $130-150$ \\
\hline
\end{tabular}

viceversa quello delle basi sugli acidi. Studi dietetici in diverse popolazioni occidentali hanno osservato che il carico dietetico netto di acidi è maggiore nei pazienti calcolotici rispetto ai controlli sani. ${ }^{16-19}$ Gli studi italiani hanno osservato come l'eccessivo carico acido dei calcolotici sia attribuibile ad un inadeguato consumo di vegetali e quindi ad uno scarso apporto di basi, mentre gli studi statunitensi lo hanno spiegato con un eccessivo consumo di carni. ${ }^{16-19}$ Quindi l'aumento del carico netto di acidi può essere causato da abitudini alimentari scorrette che conducono ad un inappropriato rapporto nutrizionale tra carni e vegetali. L'elevato carico netto di acidi può predisporre alla calcolosi renale agendo sulla acidificazione urinaria, sulla escrezione del citrato e del calcio e sulla precipitazione dell'acido urico e del calcio-fosfato.

Quanto abbiamo descritto sottolinea l'importanza della dieta per la prevenzione della calcolosi renale. Tuttavia gli studi condotti fino ad oggi non spiegano il percorso patogenetico attraverso il quale i singoli nutrienti possono indurre lo sviluppo di calcolosi renale. In questo contesto non possiamo escludere che fattori alimenti non ancora noti possano esercitare un ruolo litogenetico. ${ }^{20}$

\section{I fattori predisponenti alla recidiva di calcolosi}

Quando i pazienti sono esposti a specifici fattori che amplificano il rischio di recidiva di calcolosi, i provvedimenti appena descritti devono essere associati ad una terapia farmacologica (figura 1). I fattori di rischio sono stati stabiliti nelle linee guida dell'EAU ${ }^{2}$ e sono riportati nella Tabella 3: comprendono la storia clinica, difetti metabolici acquisiti, malattie monogeniche, farmaci, le malformazioni e le infezioni urinarie. La decisione di intraprendere una terapia farmacologica si basa inoltre sulla composizione dei calcoli. ${ }^{21}$ I fattori di rischio elencati nella Tabella 3 non forniscono tuttavia al clinico tutti gli elementi necessari per un giudizio sulla necessità di terapia. Un giudizio in tal senso non può fare a meno di valutare anche l'attività della malattia e le anomalie metaboliche urinarie ad essa associate. Non esiste un criterio condiviso che valuti l'attività clinica della malattia calcolotica, ma sulla base di quanto riportato nella letteratura medica ${ }^{22-26}$ potremmo considerare attiva la calcolosi che abbia prodotto almeno tre calcoli negli ultimi cinque anni, dei quali uno per anno negli ultimi due anni. Misurare l'escrezione delle sostanze litogene contribuisce a farci capire la tendenza dei pazienti calcolotici alla recidiva. Studi prospettici in tal senso sono però stati condotti solo per l'ipercalciuria che è presente nel $30-50 \%$ dei pazienti. Una percentuale minore di pazienti è interessata da iperuricuria, iperossaluria o ipocitraturia. Oltre a questi parametri, l'escrezione di fosfato, potassio, magnesio, urea, cloro e sodio nelle urine ci informa sulla quantità di proteine (fosfato, urato ed urea), vegetali (potassio e magnesio) e sale da cucina (sodio e cloro) introdotti nelle 24 ore; inoltre il cloro urinario consente il computo del gap anionico urinario che viene calcolato come differenza tra il cloro urinario e la somma 


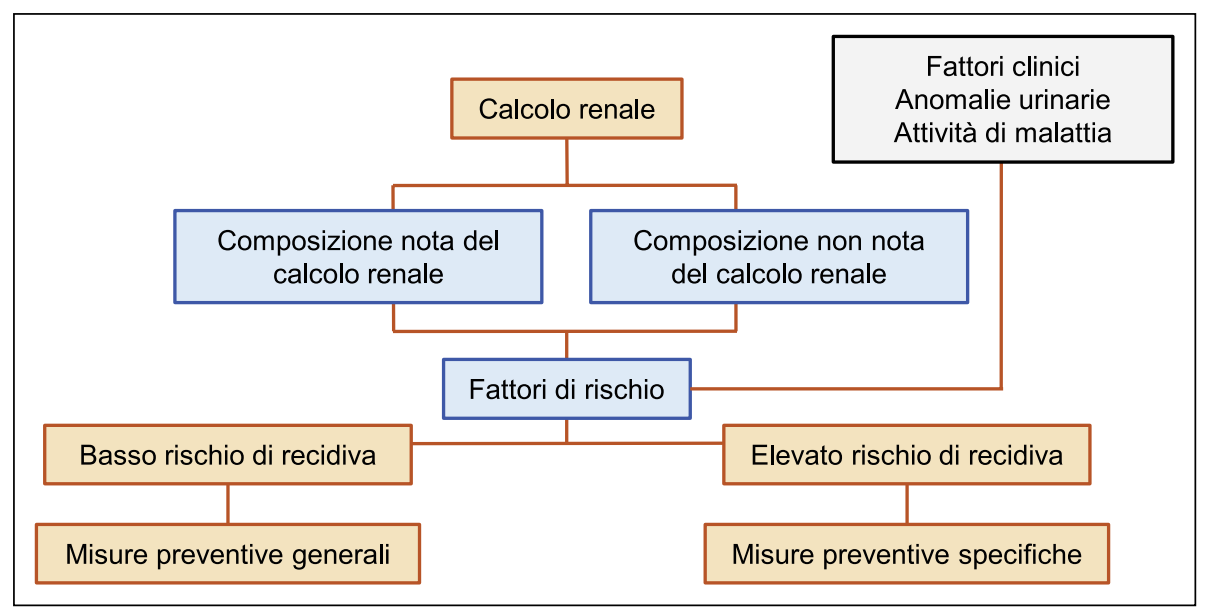

Figura I. Schema dell'approccio clinico ai pazienti calcolotici, tra i quali devono essere distinti i pazienti ad alto o basso rischio di recidiva sulla base della composizione dei calcoli, della storia clinica, delle anomalie urinarie e della attività di malattia. La tabella 3 elenca i fattori clinici di rischio di recidiva.

del sodio e del potassio urinari e che stima l'ammoniogenesi. Questi dosaggi sono utili per guidare il clinico nella prescrizione dietetica e farmacologica e anche per definire la compliance terapeutica del paziente.

L'analisi dei fattori di rischio, dell'attività clinica della malattia calcolotica e delle anomalie urinarie ci consente di individuare due gruppi di pazienti. Il primo gruppo comprende i pazienti privi di fattori di rischio e che soffrono di una calcolosi a scarsa attività clinica; questi necessitano delle sole misure preventive generali. Il secondo gruppo comprende i pazienti che presentano uno $o$ più fattori di rischio e/o malattia calcolotica attiva e che perciò si giovano di provvedimenti farmacologici specifici che vanno ad aggiungersi a quelli generali.

\section{Le misure preventive specifiche}

\section{Terapia della calcolosi idiopatica di calcio}

La terapia della calcolosi di calcio viene indirizzata dalla composizione dei calcoli e dalle anomalie urinarie emerse nello screening metabolico dei pazienti (tabella 4).

L'ipercalciuria trova nei diuretici tiazidici il trattamento più specifico, che deve essere quasi sempre accompagnato dalla somministrazione di un sale alcalino di potassio (come il citrato di potassio) allo scopo di compensare le perdite di potassio e la riduzione del citrato urinario indotte dal diuretico. L'efficacia antilitogena del tiazidico è stata confermata in diversi studi randomizzati e controllati, anche se tutti di piccole dimensioni. ${ }^{25-29}$ L'azione antilitogena dei tiazidici (figura 2) è probabilmente legata al loro effetto di riduzione della calciuria. Il tiazidico inibisce il cotrasporto cloro-sodio (NCC) della membrana luminale nelle cellule del dotto convoluto distale; conseguentemente riduce il sodio intracellulare, attivando così lo scambiatore sodiocalcio (NCX1) posto sulla membrana basolaterale di queste cellule; l'attivazione di NCX1 aumenta il trasporto del calcio verso l'interstizio ed il riassorbimento di calcio. La somministrazione dei tiazidici aumenta anche l'espressione dei canali del calcio (TRPV5) della membrana apicale delle cellule del dotto convoluto distale; questo canale media l'influsso di calcio dal lume tubulare contribuendo al riassorbimento di calcio. ${ }^{30}$ Per avere un significativo effetto sulla calciuria, idroclorotiazide o clortalidone devono essere somministrati alla dose di almeno $25 \mathrm{mg}$ die; inoltre il clortalidone sembra ottenere un maggiore effetto rispetto all'idroclorotiazide. ${ }^{31}$ Oltre a queste due molecole, anche l'indapamide $(2,5 \mathrm{mg} / \mathrm{die})$ può essere usata allo scopo di limitare la calciuria e proteggere dalla calcolosi.

L'ipocitraturia può essere corretta dalla somministrazione di sali alcalini quali il bicarbonato di sodio, il citrato di potassio o il citrato di potassio e magnesio. Il citrato urinario è efficace nella prevenzione delle recidive di calcolosi perché inibisce l'aggregazione e la crescita dei cristalli di calcio-ossalato, ma anche dei cristalli di fosfato di calcio. ${ }^{31}$ Oltre ad aumentare l'escrezione di citrato, queste sostanze alcalinizzano le urine favorendo così la solubilizzazione dell' acido urico e la scomparsa del suo effetto epitattico sui cristalli di calcio ossalato. Diversi trial randomizzati e controllati di piccole dimensioni campionarie hanno dimostrato l'efficacia del citrato di potassio nel prevenire la recidiva di calcolosi; 22 , 24-26 nessuno di questi trial ha distinto tra pazienti con calcoli di ossalato o fosfato di calcio; inoltre in un trial con risultati negativi circa l'efficacia antilitogena del citrato, 16 pazienti trattati con citrato avevano recidivato nel corso del follow-up, ma solo uno di questi pazienti aveva calcoli di fosfato di calcio. ${ }^{23}$ Queste considerazioni ed $\mathrm{i}$ risultati ottenuti in vitro dimostrano come la prevenzione con citrato di potassio possa essere somministrata sia ai pazienti con calcoli di calcio-ossalato, 
Tabella 3. Fattori di rischio di calcolosi renale (modificati dalle linee guida della EAU). ${ }^{2}$

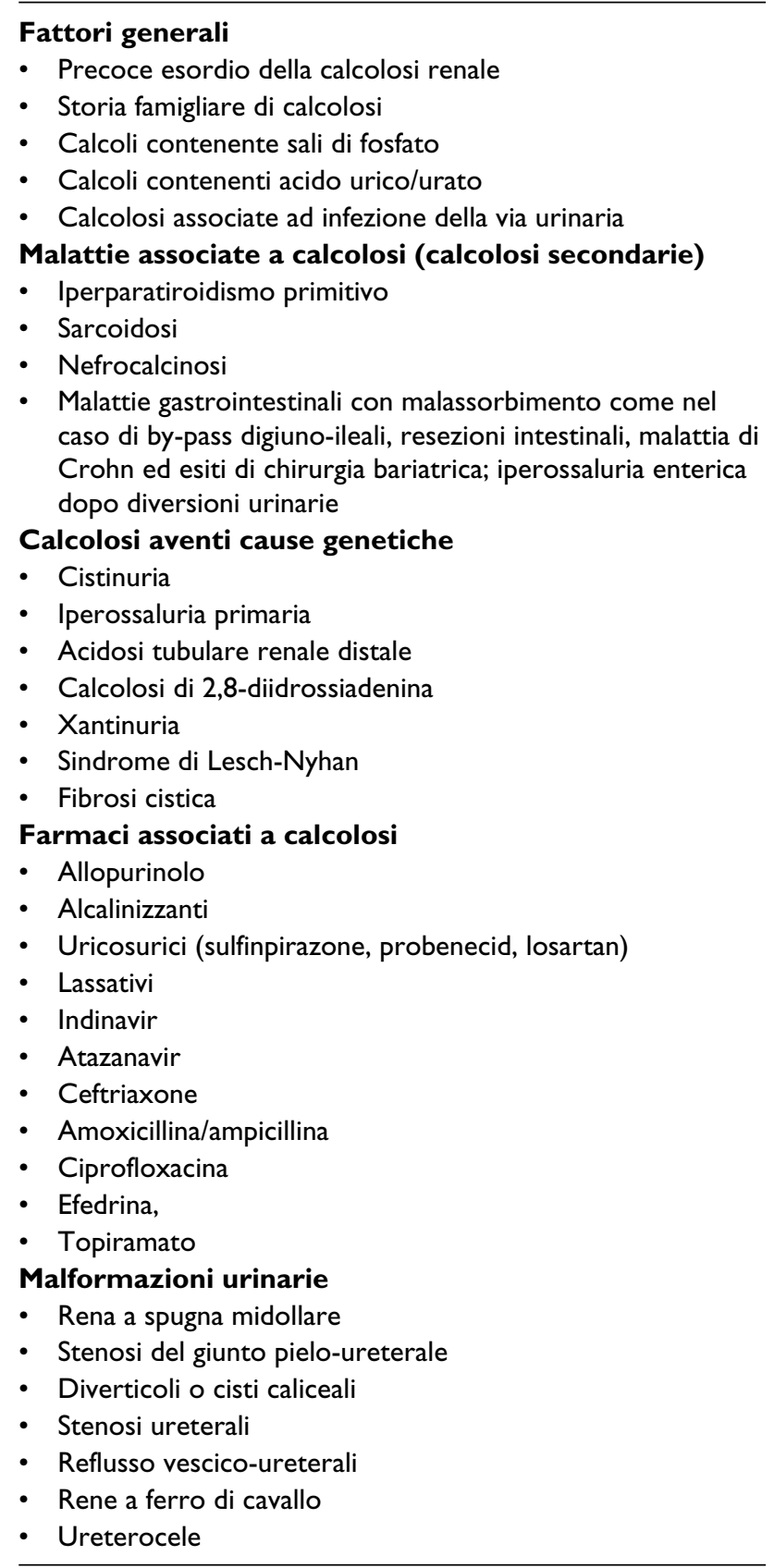

che ai pazienti con calcoli di calcio-fosfato: l'effetto antilitogeno del citrato è legato alla inibizione dello sviluppo dei cristalli di calcio-fosfato e ossalato e sembra prevalere sull'ipotetico effetto "pro-litogeno" conseguente alla precipitazione del calcio-fosfato in urine alcaline. La terapia con alcali usa 3-9 g/die di citrato di potassio o di potassio e magnesio divisi in 2-3 somministrazioni; essa però non riesce a modificare $\mathrm{i}$ valori della citraturia nel 20-30\% dei pazienti ipocitraturici, verosimilmente per un deficit di assorbimento intestinale delle basi. ${ }^{32}$ In alternativa al citrato, il bicarbonato di sodio viene somministrato alla dose di 1,5-9 g/die in 2-4 somministrazioni.

L'iperuricuria predispone sia alla calcolosi uratica che di calcio ed è perciò bersaglio della terapia in entrambi i tipi di calcolosi. Può essere trattata con citrato di potassio o sodio bicarbonato alle dosi già riportate per mantenere il $\mathrm{pH}$ delle urine tra 6.5 e 7 ed impedire la cristallizzazione dell'acido urico. Può però essere corretta anche con basse dosi di allopurinolo oppure febuxostat (100 mg die o 40 $\mathrm{mg}$ die, rispettivamente), lasciando le dosi piene (300 mg o $80 \mathrm{mg}$, rispettivamente) ai pazienti con iperuricemia e gotta. ${ }^{33}$ L'acido urico come è noto deriva dal catabolismo delle basi puriniche del DNA (adenina e guanina) e i due farmaci citati inibiscono l'attività dell'enzima xantinoossidasi che catalizza l'ossidazione della xantina in acido urico come ultimo passaggio del catabolismo delle basi puriniche. La xantina viene escreta con le urine e può for mare raramente calcoli renali.

L'iperossaluria si manifesta per escrezioni di ossalato superiori a $40 \mathrm{mg} / \mathrm{die}$. Le forme più gravi si presentano con ossaluria oltre i $100 \mathrm{mg} / \mathrm{die}$ e sono distinte in forme primarie monogeniche causate da una mutazione di uno dei 3 geni che codificano per enzimi epatici che metabolizzano l'ossalato (alanina-gliossilatoaminotrasferasi, gliossilato reduttasi, idrossi-ossoglutarato aldolasi), e forme enteriche causate da un iperassobimento intestinale di ossalato che si verifica nei pazienti con malassorbimento, resezioni o bypass intestinali. Nelle calcolosi idiopatiche, l'iperossaluria si pone tra 40 e 100 $\mathrm{mg} / \mathrm{die}$ e non sono chiare le sue cause. Il trattamento di questo difetto utilizza gli stessi farmaci impiegati nelle forme primarie o enteriche: la piridossina, il calcio e il citrato di potassio. La piridossina è un cofattore che attiva l'enzima alanina/gliossilato aminotransferasi il cui gene è mutato nella iperossaluria primaria di tipo $1^{\circ}$ e che spiega l' $80 \%$ dei casi di iperossaluria primitiva. L'enzima alanine/ gliossilato aminotransferasi catalizza la transaminazione di alanina e gliossilato a formare glicina e piruvato; in alternativa a questa via il gliossilato viene metabolizzato a ossalato. ${ }^{34}$ Perciò la piridossina sottrae il gliossilato alla produzione dell'ossalato. Il calcio viene viceversa usato nelle forme di iperossaluria enterica perché lega l'ossalato nel lume intestinale impedendone l'assorbimento. Il citrato o il bicarbonato possono essere associati a queste terapie per impedire la cristallizzazione urinaria dell'ossalato e per combattere l'acidosi metabolica e l'ipopotassiemia spesso presenti nei pazienti con malassorbimento. Alla somministrazione di questi farmaci bisogna associare la dieta idropinica, priva delle verdure più ricche di ossalato come gli spinaci, le bietole, il cavolo e la soia (tabella 5) e con un ridotto apporto di grassi perché gli acidi grassi legano il calcio nel lume intestinale ed aumentano la disponibilità dell'ossalato per l'assorbimento. All'opposto, la frutta e i pomodori non causano un carico di ossalato. ${ }^{35}$ Oltre alle verdure una parte della produzione endogena di 
Tabella 4. Principali alterazioni urinarie riscontrabili nei pazienti con la calcolosi renale di calcio e la loro terapia.

\begin{tabular}{|c|c|c|}
\hline Alterazione & Limite & Terapia specifica \\
\hline \multirow[t]{3}{*}{ Ipercalciuria } & uomini $>7,5 \mathrm{mmol} / \mathrm{die}(300 \mathrm{mg} / \mathrm{die})$ & Idroclorotiazide $25-50 \mathrm{mg}$ die (in $\mathrm{I}-2$ somministrazioni die) \\
\hline & donne $>6,25 \mathrm{mmol} / \mathrm{die}(250 \mathrm{mg} / \mathrm{die})$ & Clortalidone $25-50 \mathrm{mg}$ die (in I-2 somministrazioni die) \\
\hline & & Indapamide 2,5 mg/die (una somministrazione die) \\
\hline \multirow[t]{2}{*}{ Ipocitraturia } & uomini $>1,9 \mathrm{mmol} / \mathrm{die}(360 \mathrm{mg} / \mathrm{die})$ & Citrato di potassio 3-9 g/die (in 2-3 somministrazioni) \\
\hline & donne $>$ I,7 mmol/die (320 mg/die) & Sodio bicarbonato (in 3-4 somministrazioni) \\
\hline Iperossaluria & $>44 \mu \mathrm{mol} / \mathrm{die}(40 \mathrm{mg} / \mathrm{die})$ & $\begin{array}{l}\text { Piridossina } 5-10 \mathrm{mg} / \mathrm{kg} \text { die (una volta al giorno) } \\
\text { Calcio } 500-1000 \mathrm{mg} \text { (forme enteriche) }\end{array}$ \\
\hline \multirow[t]{3}{*}{ Iperuricuria } & $>4 \mathrm{mmol} / \mathrm{die}(670 \mathrm{mg} / \mathrm{die})$ & Citrato di potassio $3-9 \mathrm{~g} / \mathrm{die}$ (in 2-3 somministrazioni) \\
\hline & & Sodio bicarbonato (in 3-4 somministrazioni) \\
\hline & & Allopurinolo $100-300 \mathrm{mg}$ die (una volta al giorno) \\
\hline Ipomagnesiuria & $>3 \mathrm{mmol} / \mathrm{die}(70 \mathrm{mg} / \mathrm{die})$ & Ossido di magnesio 1,5 g/die (in 3 somministrazioni) \\
\hline
\end{tabular}

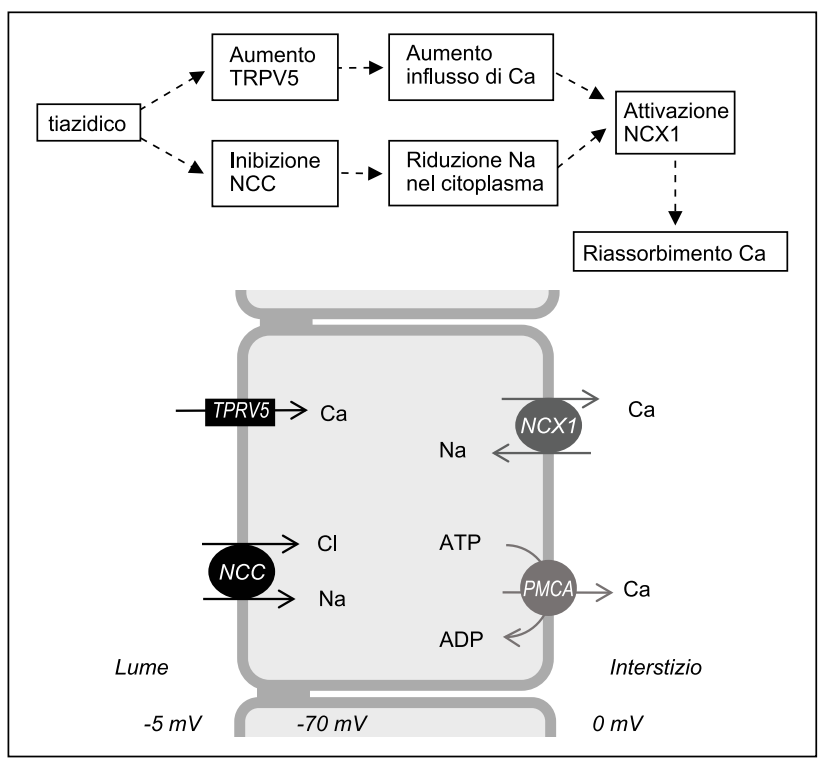

Figura 2. Effetto dei tiazidici sul trasporto del calcio nel tubulo contorto distale.

ossalato deriva dalla vitamina $\mathrm{C}$, viene perciò raccomandato di non superare i $150 \mathrm{mg}$ di apporto giornaliero. ${ }^{36}$

L'ipomagnesiuria è una poco frequente condizione che predispone alla precipitazione dei sali di calcio e che può essere compensata dalla somministrazione di ossido di magnesio (500 mg tre volte die) $)^{37}$. La terapia con ossido di magnesio rappresenta anche una valida alternativa alle terapie sino ad ora menzionate per la calcolosi renale di calcio. Una limitazione all'utilizzo dell'ossido di magnesio è la frequente comparsa di diarrea.

Abbiamo messo in evidenza come l'alcalinizzazione delle urine possa essere utilizzata per prevenire la precipitazione dell'acido urico. Le linee guida EAU propongono anche di utilizzare sostanze che acidificano le urine nella terapia della calcolosi renale di fosfato e da infezione. ${ }^{2}$ Le sostanze che possono essere usate per acidificare le urine sono il cloruro di ammonio e la metionina. Il cloruro di ammonio può essere somministrato come prodotto galenico alla dose di $0.1 \mathrm{~g} / \mathrm{kg}$ al giorno in 3-4 somministrazioni. La metionina è un amminoacido con gruppi solforici acidi che può essere prescritto alla dose di $500-1500 \mathrm{mg} / \mathrm{die}$ in tre somministrazioni. La terapia acidificante non viene però proposta dalle linee guida americane ed italiane a cause delle controindicazioni al suo utilizzo. L'uso di sostanze acidificanti può provocare acidosi soprattutto nei pazienti con insufficienza renale e con pielonefrite cronica associata a calcolosi infetta. Inoltre, l'elevato carico acido contribuisce a ridurre l'escrezione di citrato, potrebbe accrescere il rischio di calcolosi acido urico e potrebbe favorire la riduzione della massa ossea trovando nei sali contenuti nell'osso un tampone a cui legarsi liberando calcio.

\section{Terapia della calcolosi di urato}

L'eccessiva acidità delle urine e la iperescrezione di acido urico sono i fattori patogenetici della calcolosi di acido urico (tabella 6). L'insulino-resistenza potrebbe essere coinvolta nella eccessiva acidificazione delle urine perché impedisce all'insulina di stimolare lo scambiatore sodioidrogeno (NHE3) delle cellule del tubulo prossimale che trasporta ammonio $\left(\mathrm{NH}_{4}\right)$ nel lume tubulare. I pazienti insulino-resistenti potrebbero perciò avere una riduzione della ammoniogenesi ${ }^{38}$ e della capacità delle urine di tamponare gli ioni idrogeno nelle urine con conseguente riduzione del $\mathrm{pH}$ urinario. I sali alcalini di bicarbonato o di citrato sono perciò un componente importante del trattamento dei pazienti con calcoli di acido urico al quale possono essere associati l'allopurinolo o il febuxostat a basse dosi oppure a dosi piene quando è presente iperuricemia. ${ }^{39}$

\section{Terapia della calcolosi di cistina}

La cistinuria è una malattia ereditaria autosomica recessiva, causata de una mutazione nei geni SLC3A1 (cromosoma 2p21) e SLC7A9 (cromosoma 19q13.11). SLC3A1 codifica 
Tabella 5. Contenuto di ossalato dei alcuni vegetali espresso in milligrammi per $100 \mathrm{~g}$ di alimento (Kohman EF. Oxalic acid in foods and its behavior and fate in the diet. J Nutr 1939; 18: 233-246).

\begin{tabular}{lll}
\hline Tipo di alimento & Alimento & Contenuto di ossalato (mg/l00 g) \\
\hline Verdure a foglia & Prezzemolo & 1700 \\
& Erba cipollina & 1480 \\
& Spinaci & 970 \\
& Bietole, foglie & 916 \\
& Rabarbaro & 870 \\
& Barbabietola rossa & 870 \\
& Cavolo, foglie & 660 \\
& Patata & 56 \\
& Indivia & 27 \\
& Cipolla & 23 \\
Altri frutti e vegetali & Mandorle & 430 \\
& Cioccolato fondente, cacao & $650-780$ \\
& Tofu & $140-280$ \\
& Noccioline & 187 \\
& Aime & 110 \\
& Arance & 24 \\
\hline
\end{tabular}

Tabella 6. Criteri e modalità di trattamento delle calcolosi di urato e cistina e delle calcolosi da infezione.

\begin{tabular}{|c|c|c|}
\hline Calcolo & Tipo di terapia & Posologia e criteri \\
\hline \multirow[t]{3}{*}{ Urato } & Alcalinizzazione urinaria & Mantenere il pH urine tra 6,2 e 6,8 \\
\hline & Antiuricosurici & Allopurinolo $100 \mathrm{mg}$ die o Febuxostat $40 \mathrm{mg}$ in assenza di iperuricemia; \\
\hline & & Allopurinolo $150-300 \mathrm{mg}$ die o Febuxostat $80 \mathrm{mg}$ in presenza di iperuricemia \\
\hline \multirow[t]{3}{*}{ Cistina } & Aumento volume urinario & Mantenere volume urine oltre i $3 \mathrm{~L}$ \\
\hline & & $\begin{array}{l}\text { Citrato di potassio e bicarbonato di sodio controllando } \mathrm{pH} \text { urine ad ogni } \\
\text { minzione nelle prime fasi della terapia per mantenere } \mathrm{pH}>7,5\end{array}$ \\
\hline & Complessare la cistina urinaria & Se non bastano le precedenti misure trattare con tiopronina $500-2000 \mathrm{mg}$ die \\
\hline \multirow[t]{5}{*}{ Da infezione } & Bonifica chirurgica & Deve essere il più possibile completa \\
\hline & Terapia antibiotica & Somministrare antibiotico a cicli brevi o lunghi \\
\hline & Acidificazione urinaria & Cloruro di ammonio I g 2 o 3 volte die \\
\hline & & Metionina $200-500 \mathrm{mg} \mathrm{I}-3$ volte die \\
\hline & Inibizione ureasi & Acido acetoidrossamico $15 \mathrm{mg} / \mathrm{kg} / \mathrm{die}$ \\
\hline
\end{tabular}

per il carrier che riassorbe gli amminoacidi basici (cistina, lisina, ornitina, arginina) posto nella membrana apicale delle cellule del tubulo prossimale. SLC7A9 codifica per la proteina di ancoraggio del carrier alla membrana. Gli omozigoti per le mutazioni di questi geni sono affetti da cistinuria ed eleminano con le urine quantità di cistina superiori a $1000 \mu \mathrm{mol} / \mathrm{die}$ (normale $<400 \mu \mathrm{mol} / \mathrm{die} .{ }^{40,41} \mathrm{La}$ cistina è poco solubile in ambiente acquoso e precipita nelle urine sviluppando calcoli. La solubilità della cistina cresce con l'alcalinizzazione del mezzo di soluzione e con il volume urinario. La terapia della calcolosi cistinica consiste perciò nell'alcalinizzazione delle urine e nell'aumento del volume urinario (tabella 6). Generalmente viene consigliato a questi pazienti di mantenere il volume urinario oltre $3 \mathrm{~L}$ al giorno, mentre il $\mathrm{pH}$ urine deve essere mantenuto superiore a 7.5. Dosare la cistinuria nelle urine di 24 ore consente di fare la diagnosi, ma anche di avere un'idea del volume urinario teoricamente necessario per mantenere disciolta la cistina: nelle soluzioni a $\mathrm{pH}=7$ la cistina è solubile fino alla concentrazione di $1250 \mu \mathrm{mol} / \mathrm{L}$ $(300 \mathrm{mg} / \mathrm{L})$, in quelle a $\mathrm{pH}=7.5$ la sua solubilità sale fino a $1650 \mu \mathrm{mol} / \mathrm{L} \quad(400 \mu \mathrm{mg} / \mathrm{L})$, per poi crescere esponenzialmente a $\mathrm{pH}$ ancora più alti. Nelle fasi di impostazione della terapia il paziente deve progressivamente aumentare le dosi di alcali e controllare il $\mathrm{pH}$ delle urine ad ogni minzione, in modo da essere certi che sia stabilmente superiore a 7,5. Le urine possono essere alcalinizzate somministrando citrato di potassio (4-9 $\mathrm{g}$ divisi in tre somministrazioni al giorno, l'ultima delle quali prima di coricarsi) o bicarbonato di sodio (2-9 $\mathrm{g}$ divisi in 3-4 somministrazioni al giorno, l'ultima delle quali prima di coricarsi), che possono essere anche 
combinati insieme per evitare un eccessivo carico di potassio. In alternativa può essere utilizzata l' acetazolamide (250 mg 1-2 volte die) che però per la sua azione sul riassorbimento di bicarbonato provoca acidosi sistemica. Questi farmaci devono ovviamente essere usati con attenzione nei pazienti con insufficienza renale. Oltre a questi provvedimenti possono essere utili anche la dieta iposodica, che riducendo l'escrezione di sodio diminuisce anche quella di cistina, e povera di metionina, amminoacido precursore della cistina. La dieta povera di metionina è però di difficile realizzazione perché prevede di ridurre il consumo di cibi proteici, quali carni, latticini e uova. Se con queste indicazioni sono insufficienti, possono essere impiegati farmaci come la tiopronina $(0,5-2 \mathrm{~g} / \mathrm{die})$ che lega la cistina nelle urine e riduce il rischio della sua precipitazione. La somministrazione di tiopronina deve comunque essere associata al mantenimento di un flusso urinario alcalino e di volume elevato e può essere poco tollerata soprattutto a causa dei disturbi gastrici e digestivi che può provocare.

\section{Terapia della calcolosi da infezione}

Sicuramente quella associata a infezione è la forma più complessa e severa di calcolosi perché l'efficacia della cura presuppone di coordinare il trattamento chirurgico con quello medico e perché l'infezione tende a cronicizzare compromettendo la funzione renale. ${ }^{42}$ L'eliminazione completa dei calcoli con i metodi chirurgici è perciò necessaria per bonificare l'ambiente urinario ed evitare le complicanze. A questo si associa la terapia antibiotica che può essere somministrata con diverse strategie (cicli brevi o lunghi). È inoltre disponibile un inibitore della ureasi batterica come l'acido acetoidrossamico che in trial randomizzati si è dimostrato efficace per inibire la crescita dei calcoli associati ad infezione da germi ureasi produttori; questo farmaco non è però disponibile in Italia. ${ }^{43} \mathrm{I}$ pazienti con infezioni da germi ureasi produttori hanno urine alcaline perché l'ureasi batterica scinde l'urea in ossido di carbonio e ammoniaca $\left(\mathrm{NH}_{3}\right)$ dalla quale si forma ammonio $\left(\mathrm{NH}_{4}\right)$; questo processo alcalinizza le urine e induce la formazione di ioni usualmente non presenti che aumentano ulteriormente il valore del $\mathrm{pH}$ urinario. Come già riportato, le linee guida EAU propongono l'acidificazione urinaria come metodo per combattere la calcolosi infetta, ${ }^{44}$ tuttavia il processo di alcalinizzazione descritto nelle infezioni da germi ureasi produttori è di tale entità che difficilmente riesce ad essere corretto dalla terapia acidificante. Inoltre, la somministrazione di sostanze acidificanti può provocare acidosi, specie nei pazienti con insufficienza renale e pielonefrite cronica. Come già riferito il cloruro di ammonio $(0,1 \mathrm{~g} / \mathrm{kg}$ al giorno divisi in $3-4$ somministrazioni/ die) e la metionina $(500-1500 \mathrm{mg} / \mathrm{die}$ in tre somministrazioni) sono le sostanze acidificanti proposte (tabella 6).

\section{Conclusioni}

Abbiamo cercato di fare il punto sulla terapia di una malattia complessa come la calcolosi renale prendendo spunto dalle recenti linee guida e dalla pratica clinica. Appare evidente da quanto scritto che la dieta copre un ampio spazio terapeutico e che questo spazio è stato progressivamente affinato negli ultimi 50 anni. Attraverso provvedimenti generali e misure terapeutiche specifiche sono state definite linee terapeutiche che possono essere applicate alle diverse situazioni cliniche e alle diverse condizioni predisponenti. Fa eccezione la calcolosi da infezione, che ha una linea terapeutica poco definita, sia per la carenza degli strumenti che per la difficoltà d'intervento. Le linee terapeutiche tracciate considerano " $i$ soliti farmaci" già da molto tempo usati per la calcolosi renale; non si intravedono infatti grandi novità terapeutiche all'orizzonte: il tiosolfato di sodio sembra essere efficace come antilitogeno, ma non è ancora del tutto chiara la sua attività, ${ }^{45}$ gli acidi grassi polinsaturi sono stati testati negli scorsi anni in piccoli studi, ma non hanno mai avuto grande richiamo, ${ }^{46}$ l'uso dei batteri che metabolizzano l'ossalato nel colon è stato per ora solo ipotizzato. ${ }^{35}$

Quello che però emerge dalle diverse linee guida e dalla pratica quotidiana, è la necessità di identificare le caratteristiche dei singoli pazienti per proporre la terapia più idonea. Questo processo di analisi clinica ed anamnestica prende tempo e non è di facile applicazione nei nostri ambulatori, tuttavia è l'approccio più corretto ed esistono studi che hanno certificato la maggiore efficacia del trattamento antilitogeno personalizzato rispetto ai trattamenti che impiegano prescrizioni standard. ${ }^{47}$ Questa impostazione comporta però la crescita di un nuovo soggetto clinico che sappia analizzare i pazienti dal punto di vista alimentare e metabolico per trarre gli utili indirizzi terapeutici.

\section{Dichiarazione di assenza di conflitto di interessi}

Gli Autori dichiarano di non avere conflitti di interessi.

\section{Finanziamenti}

Gli Autori dichiarano di non aver ricevuto finanziamenti specifici da qualsiasi ente nei settori pubblico, privato o senza fini di lucro.

\section{Bibliografia}

1. Gruppo di Studio Multidisciplinare per la Calcolosi Renale. Percorso diagnostico-terapeutico per il paziente con calcolosi urinaria. G Ital Nefrol 2010; 27: 282-289.

2. Skolarikos A, Straub M, Knoll T, et al. Metabolic Evaluation and Recurrence Prevention for Urinary Stone Patients: EAU Guidelines. Eur Urol 2015; 67: 750-763.

3. Pearle MS, Goldfarb DS, Assimos DG, et al. Medical Management of Kidney Stones: AUA Guideline. J Urol 2014; 192: 316-324.

4. Qaseem A, Dallas P, Forciea MA, et al. Clinical Guidelines Committee of the American College of Physicians. Dietary and pharmacologic management to prevent recurrent 
nephrolithiasis in adults: A clinical practice guideline from the American College of Physicians. Ann Intern Med 2014; 161: 659-667.

5. Wang CJ, Grantham JJ and Wetmore JB. The medicinal use of water in renal disease. Kidney Int 2013; 84: 4553.

6. Ticinesi A, Nouvenne A, Maalouf NM, et al. Salt and nephrolithiasis. Nephrol Dial Transplant 2016; 31: 39-45.

7. Curhan GC, Willett WC, Speizer FE, et al. Comparison of dietary calcium with supplemental calcium and other nutrients as factors affecting the risk for kidney stones in women. Ann Intern Med 1997; 126: 497-504.

8. Taylor EN and Curhan GC. Dietary calcium from dairy and non-dairy sources and risk of symptomatic kidney stones. $J$ Urol 2013; 193: 864-868.

9. Pak CY, Britton F, Peterson R, et al. Ambulatory evaluation of nephrolithiasis. Classification, clinical presentation and diagnostic criteria. Am J Med 1980; 69: 19-30.

10. Robertson WG, Heyburn PJ, Peacock M, et al. The effects of high animal protein intake on the risk of calcium stone formation in the urinary tract. Clin Sci 1979; 57: 285288.

11. Borghi L, Schianchi T, Meschi T, et al. Comparison of two diets for the prevention of recurrent stones in idiopathic hypercalciuria. N Engl J Med 2002; 346: 77-84.

12. Turney BW, Appleby PN, Reynard JN, et al. Diet and risk of kidney stones in the Oxford cohort of the European Prospective Investigation into Cancer and Nutrition (EPIC). Eur J Epidemiol 2014; 29: 363-369.

13. Maalouf NM, Moe OW, Adams-Huet B, et al. Hypercalciuria associated with high dietary protein intake is not due to acid load. J Clin Endocrinol Metab 2011; 96: 3733-4370.

14. Kerstetter JE, O'Brien KO, Caseria DM, et al. The impact of dietary protein on calcium absorption and kinetic measures of bone turnover in women. J Clin Endocrinol Metab 2005; 90: $26-31$.

15. Remer T and Manz F. Potential renal acid load of foods and its influence on urine pH. J Am Diet Assoc 1995; 95: 791-797.

16. Vezzoli G, Dogliotti E, Terranegra A, et al. Dietary style and acid load in an Italian population of calcium kidney stone formers. Nutr Metab Cardiovasc Dis 2015; 25: 588-593.

17. Trinchieri A, Maletta A, Lizzano R, et al. Potential renal acid load and the risk of renal stone formation in a caseecontrol study. Eur J Clin Nutr 2013; 67: 1077-1080.

18. Breslau N, Brinkley L, Hill KD, et al. Relationship of animal protein-rich diet to kidney stone formation and calcium metabolism. J Clin Endocrinol Metab 1988; 66: 140-146.

19. Mandel EJ, Taylor EN and Curhan GC. Dietary and lifestyle factors and medical conditions associated with urinary citrate excretion. Clin J Am Soc Nephrol 2013; 8: 901-908.

20. Calvez J, Poupin N, Chesneau C, et al. Protein intake, calcium balance and health consequences. Eur J Clin Nutr 2012; 66: 281-295.

21. Tiselius HG, Daudon M, Thomas K, et al. Metabolic work-up of patients with urolithiasis: indications and diagnostic algorithm. Eur Urol Focus 2017; 3: 61-72.

22. Barcelo P, Wuhl O, Servitge E, et al. Randomized doubleblind study of potassium citrate in idiopathic hypocitraturic calcium nephrolithiasis. J Urol 1993; 150: 1761-1764.
23. Hofbauer J, Höbarth K, Szabo N, et al. Alkali citrate prophylaxis in idiopathic recurrent calcium oxalate urolithiasis - a prospective randomized study. $\mathrm{Br} J$ Urol 1994; 73: 362-365.

24. Ettinger B, Pak CY, Citron JT, et al. Potassium-magnesium citrate is an effective prophylaxis against recurrent calcium oxalate nephrolithiasis. J Urol 1997; 158: 2069-2073.

25. Laerum $\mathrm{S}$ e and Larsen S. Thiazide prophylaxis of urolithiasis. A double-blind study in general practice. Acta Med Scand 1984; 215: 383-389.

26. Scholz K, Schwille PO and Sigel A. Double-blind study with thiazide in recurrent calcium nephrolithiasis. J Urol 1982; 128: 903-907.

27. Ohkawa M, Tokunaga S, Nakashima T, et al. Thiazide treatment for calcium urolithiasis in patients with idiopathic hypercalciuria. Br J Urol 1992; 69: 571-576.

28. Ettinger B, Citron JT, Livermore B, et al. Chlorthalidone reduces calcium oxalate calculous recurrence but magnesium hydroxide does not. J Urol 1988; 139: 679-684.

29. Ettinger B, Tang A, Citron JT, Livermore B and Williams T. Randomized trial of allopurinol in the prevention of calcium oxalate calculi. N Engl J Med 1986; 315: 1386-1389.

30. Reilly RF and Huang CL. The mechanism of hypocalciuria with $\mathrm{NaCl}$ cotransporter inhibition. Nat Rev Nephrol 2011; 7: 669-674.

31. Wolfgram DF, Gundu V, Astor BC, et al. Hydrochlorothiazide compared to chlorthalidone in reduction of urinary calcium in patients with kidney stones. Urolithiasis 2013; 41: 315-322.

32. Tiselius HG, Berg C, Fornander AM, et al. Effects of citrate on the different phases of calcium oxalate crystallization. Scanning Microsc 1993; 7: 381-390.

33. Goldfarb DS, MacDonald PA, Gunawardhana L, et al. Randomized controlled trial of febuxostat versus allopurinol or placebo in individuals with higher urinary uric acid excretion and calcium stones. Clin J Am Soc Nephrol 2013; 8: 1960-1967.

34. Cochat P and Rumsby G. Primary Hyperoxaluria. $N$ Engl J Med 2013; 369: 649-658.

35. Holmes RP, Knight $\mathrm{J}$ and Assimos DG. Lowering urinary oxalate excretion to decrease calcium oxalate stone disease. Urolithiasis 2016; 44: 27-32.

36. Thomas LDK, Elinder CG, Tiselius HG, et al. Ascorbic acid supplements and kidney stone incidence among men: A prospective study. JAMA Intern Med 2013; 173: 386-388.

37. Johansson G, Backman U, Danielson BG, et al. Effects of magnesium hydroxide in renal stone disease. J Am Coll Nutr 1982; 1: 179-185.

38. Sakhaee K, Adams-Huet B, Moe OW and Pak CY. Pathophysiologic basis for normouricosuric uric acid nephrolithiasis. Kidney Int 2002; 62: 971-979.

39. Heilberg IP. Treatment of patients with uric acid stones. Urolithiasis 2016; 44: 57-63.

40. Chillaron J, Font-Llitjos M, Fort J, et al. Pathophysiology and treatment of cystinuria. Nat Rev Nephrol 2010; 6: 424-434.

41. Goldfarb DS. Potential pharmacologic treatments for cystinuria and for calcium stones associated with hyperuricosuria. Clin J Am Soc Nephrol 2011; 6: 2093-2097.

42. Bichler KH, Eipper E, Naber K, et al. Urinary infection stones. Int J Antimicrob Agents 2002; 19: 488-498. 
43. Williams JJ, Rodman JS and Peterson CM. A randomized double-blind study of acetohydroxamic acid in struvite nephrolithiasis. N Engl J Med 1984; 311: 760-764.

44. Wall I and Tiselius HG. Long-term acidification of urine in patients treated for infected renal stones. Urol Int 1990; 45: 336-341.

45. Okonkwo OW, Batwara R, Granja I, et al. A pilot study of the effect of sodium thiosulfate on urinary lithogenicity and associated metabolic acid load in non-stone formers and stone formers with hypercalciuria. PLoS ONE 2013; 8: e60380.

46. Siener R, Jansen B, Watzer B, et al. Effect of n-3 fatty acid supplementation on urinary risk factors for calcium oxalate stone formation. J Urol 2011; 185: 719-724.

47. Kocvara R, Plasgura P, Petrik A, et al. A prospective study of nonmedical prophylaxis after a first kidney stone. $B J U$ Int 1999; 84: 393-398. 